\title{
Chirality manifestation in elastic coupling between the layers of double-walled carbon nanotubes
}

Received 00th January 20xx, Accepted 00th January 20xx

DOI: $10.1039 / \times 0 \times x 00000 x$

\author{
Sergei Rochal, ${ }^{a}$ Dmitry Levshov, ${ }^{a, b,+}$ Marina Avramenko, ${ }^{a}$ Raul Arenal, ${ }^{c, d}$ Thi Thanh Cao, ${ }^{e}$ Van Chuc \\ Nguyen, ${ }^{e}$ Jean-Louis Sauvajol ${ }^{b}$ and Matthieu Paillet ${ }^{b}$
}

\begin{abstract}
A search for new relatively easy physicochemical methods for structural identification of carbon nanotubes represents a key challenge. Here, analyzing the experimental data on double-walled carbon nanotubes (DWCNTs) obtained by us and taken from literature, we have expressed the magnitude of elastic coupling between two tubular walls forming a DWCNT as a simple function dependent not only on DWCNT diameters but also on the difference between the chirality angles of the constituent nanotubes. To get this quite unexpected result, which allows us to relate more precisely the structural parameters of a DWCNT with frequencies of its radial breathing-like modes (RBLM), we have developed a new model for the RBLM dynamics that takes into account a possible deposition of water molecules from ambient air onto the DWCNT surface. The model constructed allows us to predict the higher prevalence of DWCNTs comprising two walls with identical handedness. Application of the results obtained for identification of DWCNTs is also considered.
\end{abstract}

\section{Introduction}

Since the seminal work $^{1}$ by lijima published in 1991, carbon nanotubes (CNTs) are thought to be one of the possible successors of silicon in the post-Moore's law era. ${ }^{2-4}$ These tubular structures, conceptually formed by rolling up a graphene sheet into a cylinder, combine the high mechanical resilience and chemical stability of graphene with unique, remarkably diverse electronic and optical properties. ${ }^{5,6}$ The simplest CNTs are single-walled (SW) ones and their structure with possible chirality is characterized by a pair of non-negative integers that also strongly affect the physical and chemical SWCNT characteristics like the conductivity type (metallic or semiconducting), optical transitions energies, phonon dynamics, interaction with different molecules. ${ }^{5-7}$ In contrast to the achiral SWCNTs, the chiral ones are divided into right- and left-handed types. ${ }^{6,8}$ This handedness controls, in particular, the adsorption and orientation of the chiral molecules at the chiral tubular surface ${ }^{9,10}$ or the alignment of SWCNTs themselves on the graphite substrate during growth. ${ }^{11}$ This opens the possibility of creating unique materials and nanodevices containing along with the protein, ${ }^{12,13}$ peptide ${ }^{14-17}$ or DNA molecules, ${ }^{18,19}$ which are always specifically handed, the SWCNTs with a target handedness. In particular, using similar technology, it is possible to sort nanotubes into right-and lefthanded ones with the help of DNA molecules. ${ }^{20}$

\footnotetext{
a. Department of Nanotechnology, Faculty of Physics, Southern Federal University, 5, Zorge Street, Rostov-on-Don, 344090, Russia; E-mail: rochal_s@yahoo.fr b. Laboratoire Charles Coulomb (UMR5221), CNRS-Université de Montpellier, Montpellier, F-34095, France

c. Instituto de Nanociencia de Aragón, Campus Rio Ebro, Edificio I+D. C/Mariano Esquillo, 50018, Zaragoza, Spain

d. ARAID Foundation, 50018, Zaragoza, Spain

e. Institute of Materials Science, Vietnam Academy of Science and Technology, 18 Hoang Quoc Viet, Hanoi, Vietnam

${ }^{+}$present address: Physics Department, University of Antwerp, Universiteitsplein 1, B-2610 Antwerp, Belgium

†Electronic Supplementary Information (ESI) available: S1. Experimental data for structural characterization of individual DWCNTs S2. Some statistical information on DWCNTs; S3. Structural information for individual DWCNTs and their measured and calculated frequencies of radial breathing-like modes; S4. An example of structural identification of three DWCNTs. See DOI: 10.1039/x0xx00000x
}

The unique structure-dependent optical and electronic properties make SWCNTs fascinating materials for an extensive set of applications in photonics and nanoelectronics. ${ }^{21}$ However, being comprised of only one layer of carbon atoms, they are extremely sensitive to the local environment. ${ }^{22,23}$ This has an advantage in sensor applications, but of course makes the development of stable electronic devices more difficult. This issue can however be addressed in applications by using doublewalled carbon nanotubes (DWCNTs), consisting of two coaxial and weakly van der Waals coupled carbon layers. In DWCNTs, the inner tube possesses a special status, since the outer layer acts as a shield and thus provides higher mechanical, thermal, and chemical stability, as well as isolation from the environment. ${ }^{24-26}$

However, the structural identification of DWCNTs is not straightforward due to the fact that their properties are not just a superposition of those of the constituent SWCNTs, but can be strongly modulated by (i) interlayer van der Waals mechanical and electronic interactions, depending on the wall-to-wall distance, ${ }^{27-29}$ and (ii) Moiré interference, depending on the relative chiral angles. ${ }^{27,30,31}$ These factors combined lead to situations, where the assembly of SWCNTs with almost the same physical properties such as diameter and band gap can still result in very different DWCNTs. Therefore, DWCNTs need to be viewed as a new type of nanostructures rather than a simple superposition of two SWCNTs. 
So far, several approaches for the structure analysis of individual SWCNTS and DWCNTs have been established. They are based on high-resolution transmission electron microscopy (HRTEM), ${ }^{32}$ scanning tunneling microscopy, ${ }^{33}$ electron diffraction (ED), ${ }^{34,35}$ photoluminescence, ${ }^{36}$ Rayleigh spectroscopy, ${ }^{37}$ optical absorption, ${ }^{38}$ and resonant Raman spectroscopy. ${ }^{39-41}$ The latter approach was proved to be one of the most powerful method for the probing and indexation of carbon nanotubes. ${ }^{42,43}$ It has been extensively used for the characterization of bulk samples of individual SWCNTs on the basis of pattern recognition and comparison between experimental and calculated optical transition energies. ${ }^{40,41}$ In order to assign the structure of SWCNTs, several Raman criteria based on the features of the main Raman-active modes, namely the radial breathing modes (RBM) and $G$ modes, have been established from measurements performed on index-identified free-standing SWCNTs. ${ }^{43-45}$

Here, developing these criteria for DWCNTs, we essentially specify the dependence of radial breathing-like modes (RBLM), which manifest themselves in DWCNTs, on their structural parameters. Using the experimental data on the individual nanotubes obtained by us and other authors, we have expressed the magnitude of elastic coupling between two layers forming a DWCNT as a simple function dependent not only on the DWCNT diameters but also on the twist angle $\Delta \theta$ between the DWCNT layers. On average, for the considered sets of individual DWCNTs, the chirality-dependent contribution to the elastic coupling can be approximated as proportional to $\cos (6 \Delta \theta)$. This simple and unexpected expression is based on the symmetry consideration and on a new model proposed in order to describe the RBLM dynamics. Like the model ${ }^{46}$ valid for SWCNTs, our approach takes into account a possible deposition of water molecules (contained in the air) onto the nanotube surface and allows us to relate the structural parameters of a DWCNT with its RBLM frequencies more precisely. According to our analysis, the formation of DWCNTs consisting of the pairs of SWCNTs with the same handedness is more probable. This finding is supported by the well-known results that SWCNTs align on the graphite substrate according to their handedness ${ }^{11}$ or that the adsorption of complex chiral molecules on the SWCNT surface occurs when their handedness fits that of the tube. ${ }^{20}$ An application of the results obtained for the DWCNTs identification is also demonstrated.

\section{Results and discussion}

\section{Synthesis and preliminary analysis of DWCNTs}

Individual suspended DWCNTs were grown across open slits or commercial TEM membranes by catalytical chemical vapor deposition technique ${ }^{47}$ (Fig. 1a). Structure identification of the synthesized nanotubes was performed either by ED and HRTEM methods following the known procedures ${ }^{45,48}$ or in combination with several optical methods such as Raman and Rayleigh spectroscopies as described in Ref. 45,49 (for more details, see Methods). Fig. 1b shows an example of experimental and simulated electron diffraction patterns used to characterize individual DWCNT $(14,7) @(21,10)$ with $\Delta \theta_{h i} \approx 0.684^{\circ}$, where a

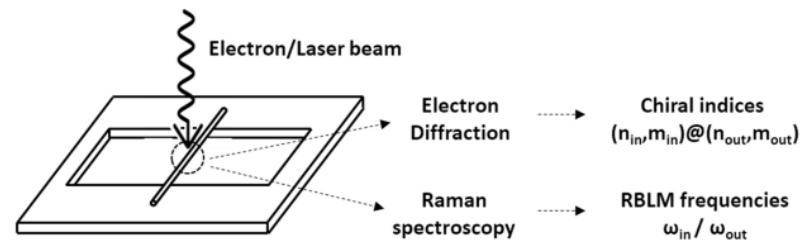

b

C

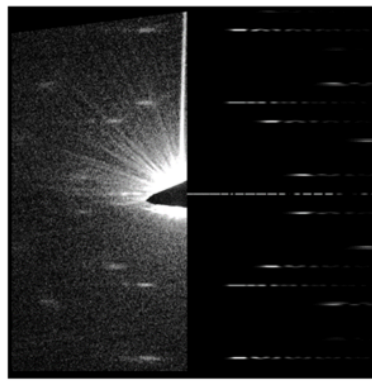

$(14,7) @(21,10)$

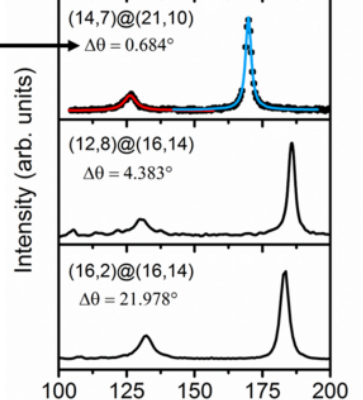

Raman shift $\left(\mathrm{cm}^{-1}\right)$

Figure 1. Experimental studies of DWCNTs. (a) Schematic illustration of the experimental procedure used in this work; (b) Experimental and simulated ED patterns of $(14,7) @(21,10)$ DWCNT; (c) RBLM range of resonance Raman spectra for $(14,7) @(21,10),(12,8) @(16,14),(16,2) @(16,14)$ DWCNTs, having close diameters and different $\Delta \theta_{h i}$.

$\Delta \theta_{\text {hi }}=|| \theta_{\text {in }}|-| \theta_{\text {out }}||$ is the handedness-invariant twist between the DWCNT layers, while $\theta_{\text {in }}$ and $\theta_{\text {out }}$ are the chiral angles of the constituent inner and outer nanotubes, respectively.

The corresponding RBLM region of Raman spectra for this DWCNT is presented in Fig. 1c. As expected for DWCNTs, two radial breathing-like modes are observed, namely in-phase (red line) and out-of-phase (blue line). ${ }^{43}$ Here we analyze new data (see Table 1) obtained on 16 individual suspended indexidentified DWCNTs. In addition, Table 1 presents the data on 8 nanotubes (with numbers $5^{45}, 8^{45}, 10^{29}, 14-17^{45}, 19^{45}$ and $22^{45}$ ) that were reported by our group earlier. See also ESI†, S1.

Model of breathing-like mode dynamics in DWCNTs with adsorbed water molecules

Despite the enormous amount of papers devoted to theoretical models of DWCNT dynamics, the first approach successfully describing the RBLM frequencies in individual DWCNTs has appeared relatively recently. ${ }^{28}$ This simple model of coupled oscillators allowed one to correlate the RBLM frequencies with those of RBMs in constituent SWCNTs. The model ${ }^{28}$ was repeatedly used and cited by other authors; in particular, it was generalized within the framework of the continuous approach, 50 which describes other low-frequency modes of DWCNTs in a simple way. Unfortunately, the original model ${ }^{28}$ predicted well the RBLM frequencies only for those DWCNTs, which had been synthesized by the same authors. In particular, an attempt ${ }^{51}$ to apply this model to the DWCNTs synthesized in our group was less successful: a quantitative match between the model predictions and the experimental RBLM frequencies in DWCNTs was not achieved, although it became clear that the dynamics of outer nanotube is modified by an environmental influence. In Ref. 45, to take this influence into account, the 
empirical dispersion law $\omega=204 / d+27$ was used for the RBM frequencies of the outer layer, but the new data obtained cannot be well explained quantitatively in this phenomenological approximation.

In Ref. 46, for the case of individual SWCNTs, it was shown that the environmental influence mainly reduces to the interaction of nanotube with a layer of water molecules adsorbed on its surface, and this layer can be effectively considered as an additional cylindrical shell. Below, in order to develop the model of RBLM dynamics in DWCNTs, we use the idea ${ }^{46}$ and restrict the continuous approach $^{50}$ to RBLM dynamics only. A Lagrangian function $L$ for such a simplified model can be expressed as

$$
L=\sum_{i=1}^{3} f_{i}+\pi H\left(C\left(u_{1}-u_{2}\right)^{2}+G\left(u_{2}-u_{3}\right)^{2}\right),
$$

where $f_{i}=2 \pi H R_{i}\left(\frac{\gamma_{i}}{2}\left(\frac{u_{i}}{R_{i}}\right)^{2}-\rho_{i} \frac{\dot{u}_{i}^{2}}{2}\right)$ are Lagrangian functions of free (non-interacting) cylindrical shells, $H$ stands for the DWCNT length, $R_{i}$ are the radii of the outer (water), middle and inner (carbon) shells; $\rho_{i}$ are shells' surface densities (for carbon nanotubes $\rho_{2}=\rho_{3}=\rho$ ); $u_{i}$ are radial displacements of the nanotubes considered; $\gamma_{1}=\gamma_{w}$ and $\gamma_{2}=\gamma_{3}=\gamma$ stand for 2D Young moduli of elasticity of water shell and graphene, respectively; $G$ is an interlayer elastic coupling in the DWCNT; $C$ describes the interaction between the deposited water molecules and the outer carbon shell and increases with its radius (provided $\rho_{1}$ is a constant). However, both $\gamma_{w}$ and $C$ parameters should tend to zero together with the surface density of water molecules $\rho_{1}$.

Solutions of the corresponding Lagrange equations $\partial L / \partial u_{i}=$ $(d / d t)\left(\partial L / \partial \dot{u}_{i}\right)$ can be obtained by substitution $u_{i}=$ $u_{i}^{0} \exp (i \omega t)$, where $t$ is time and $\omega$ is angular frequency. The resulting homogeneous linear equations may be solved if the determinant of the matrix $\mathbf{M}$,

$\mathbf{M}=\left(\begin{array}{ccc}\frac{\gamma_{w}}{R_{1}}+C-\rho_{1} R_{1} \omega^{2} & -C & 0 \\ -C & \frac{\gamma}{R_{2}}+G+C-\rho R_{2} \omega^{2} & -G \\ 0 & -G & \frac{\gamma}{R_{3}}+G-\rho R_{3} \omega^{2}\end{array}\right)$

is equal to zero. This condition determines 3 RBLM frequencies of the effectively triple-walled nanotube, comprised of a DWCNT and a water layer (on the other hand, if one substitutes $\rho_{1}=\rho$ and $\gamma_{w}=\gamma$ in matrix (2), then the equation $\operatorname{det} \mathbf{M}=0$ will easily describe the radial modes of isolated non-interacting triple-walled CNT).

Let us note that due to an environment influence the RBM frequency of SWCNT tends to increase. For instance, the interaction with the adsorbed water layer leads to the blue-shift of $5-12 \mathrm{~cm}^{-1}$ depending on the water vapor pressure. ${ }^{46}$ The $\pi-\pi$ interaction between tubes inside bundles can increase $\omega_{\text {RBM }}$ by $\sim 2-4 \mathrm{~cm}^{-1}$ (see Ref. 52), while nanotube-substrate interaction can have either negligible effect on the RBM frequency (around
$1 \mathrm{~cm}^{-1}$ as observed for index-identified SWCNT in Ref. 38) or $\sim 5-$ $7 \mathrm{~cm}^{-1}$ (estimated from Ref. 53 by comparing with 228/d relation). The effect of interlayer van-der-Waals interaction in DWCNTs is substantially more important and was reported to blue-shift RBM frequencies up to $20 \mathrm{~cm}^{-1}$ depending on the interlayer distance. ${ }^{45}$

We can thus expand the solutions of the equation $\operatorname{det} \mathbf{M}=0$ in powers of weak interaction $C$. Then, within the first order of smallness in $C$, in-phase and out-of-phase RBLM frequencies of the DWCNT with the adsorbed water molecules can be found as solutions of the following equation:

$$
M_{22} M_{33}-M_{23} M_{32}=0 .
$$

Keeping in mind that the RBM frequency of the isolated (noninteracting with environment) SWCNT satisfies an equation $\omega^{2}=$ $\gamma /\left(\rho R^{2}\right)$ (which can be simplified ${ }^{50}$ to $\omega=228 / d$, where $d$ is the nanotube diameter in $\mathrm{nm}$ and $\omega$ stands for the frequency in $\mathrm{cm}^{-1}$ ), we can rewrite Eq. (3) as

$$
\left|\begin{array}{cc}
\frac{d_{\text {out }}}{2}\left(\left(\frac{228}{d_{\text {out }}}\right)^{2}-\omega^{2}+C^{\prime}\right)+G^{\prime} & -G^{\prime} \\
-G^{\prime} & \frac{d_{\text {in }}}{2}\left(\left(\frac{228}{d_{\text {in }}}\right)^{2}-\omega^{2}\right)+G^{\prime}
\end{array}\right|=0 \text {, }
$$

where $d_{\text {out }}$ and $d_{\text {in }}$ are diameters of the outer and inner nanotubes, respectively; $G^{\prime}=G / \rho ; \quad C^{\prime}=C /\left(R_{2} \rho\right)$. The parameter $G^{\prime}$ thus determines the magnitude of interlayer coupling, while $C^{\prime}$ describes the extent to which the dispersion law of the outer nanotube is affected by the interaction with water molecules. However, note that the applicability range of Eq. (4) is not limited by the condition $C<<G$, which has been used to deduce it. In the case of $C^{\sim} G$ one can consider the coefficient $C^{\prime}$ in Eq. (4) as a way to renormalize the intrinsic RBM frequency of the outer tube, and the relation between $C^{\prime}$ and $C$ becomes nonlinear.

The values of $G^{\prime}$ and $C^{\prime}$ calculated for our DWCNTs are also given in Table 1. In order to determine $G^{\prime}$ and $C^{\prime}$, a pair of Eqs. (4) were simultaneously solved for each nanotube. In the first copy of Eq. (4) both terms $\omega^{2}$ were substituted with squared $\omega_{H}^{\text {exp }}$, in the latter one - with squared $\omega_{L}^{\text {exp }}$; the values of $\omega_{L}^{\text {exp }}$ and $\omega_{H}^{\text {exp }}$ of experimental in-phase and out-of-phase RBLM frequencies were taken from Table 1 . The obtained system was then solved in $G^{\prime}$ and $C^{\prime}$ variables and since it reduced to quadratic equation, there existed two solutions $\left\{G^{\prime}, C^{\prime}\right\}$ for every nanotube. However, we could easily choose the right solution from this couple due to the fact that both $G^{\prime}$ and $C^{\prime}$ have to be positive.

Values of $C^{\prime}$ coefficient may depend on a number of factors, for instance, on the density of the deposited water layer, its particular surface structure or even the characteristics of its dynamics. These reasons can explain the spread of $C^{\prime}$ values in the range of $2400 \ldots 3800 \mathrm{~cm}^{-2}$ (Fig. 2, blue dots). Due to this spread, two DWCNTs with exactly the same geometrical 
Table 1. Structural information and RBLM frequencies for individual DWCNTs obtained in our group. Inner $d_{\text {in }}$ and outer $d_{\text {out }}$ diameters of DWCNTs are given in nm, moduli of inner $\left|\theta_{\text {in }}\right|$ and outer $\left|\theta_{\text {out }}\right|$ chiral angles - in degrees, experimental $\omega_{L}^{\text {exp }}, \omega_{H}^{\text {exp }}$ and theoretical $\omega_{L}^{\text {theor }}, \omega_{H}^{\text {theor }}$ RBLM frequencies - in $\mathrm{cm}^{-1}$.

\begin{tabular}{|c|c|c|c|c|c|c|c|c|c|c|c|}
\hline$\#$ & DWCNT & $d_{\text {in }}$ & $\left|\theta_{i n}\right|$ & $d_{\text {out }}$ & $\left|\theta_{\text {out }}\right|$ & $\omega_{L}^{\exp }$ & $\omega_{H}^{\exp }$ & $G^{\prime}$ & $C^{\prime}$ & $\omega_{L}^{\text {theor }}$ & $\omega_{H}^{\text {theor }}$ \\
\hline 1 & $(8,4) @(18,2)$ & 0.829 & 19.11 & 1.494 & 5.21 & 171 & 288 & 2793.3 & 2673.8 & 172.2 & 290.0 \\
\hline 2 & $(7,6) @(16,6)$ & 0.882 & 27.46 & 1.542 & 15.30 & 167 & 274 & 3345.8 & 2398.3 & 167.6 & 275.1 \\
\hline 3 & $(14,1) @(21,4)$ & 1.137 & 3.42 & 1.821 & 8.57 & 146 & 215 & 3014.9 & 3053.2 & 146.1 & 215.1 \\
\hline 4 & $(14,1) @(15,12)$ & 1.137 & 3.42 & 1.834 & 26.33 & 144 & 211 & 2227.5 & 3265.4 & 143.9 & 210.9 \\
\hline 5 & $(11,6) @(18,9)$ & 1.169 & 20.36 & 1.864 & 19.11 & 141 & 206 & 2313.7 & 2884.6 & 141.7 & 207.5 \\
\hline 6 & $(14,2) @(22,4)$ & 1.182 & 6.59 & 1.898 & 8.21 & 139 & 202 & 1942.7 & 3167.9 & 138.3 & 200.7 \\
\hline 7 & $(15,2) @(23,4)$ & 1.260 & 6.18 & 1.976 & 7.89 & 134 & 190 & 1905.9 & 3039.7 & 133.8 & 189.7 \\
\hline 8 & $(10,9) @(18,11)$ & 1.289 & 28.26 & 1.985 & 22.07 & 135 & 193 & 3248.5 & 2672.6 & 134.0 & 190.3 \\
\hline 9 & $(16,2) @(16,14)$ & 1.338 & 5.82 & 2.036 & 27.80 & 132 & 183 & 2556.1 & 2987.4 & 131.9 & 182.8 \\
\hline 10 & $(12,8) @(16,14)$ & 1.365 & 23.41 & 2.036 & 27.80 & 133 & 186 & 3707.1 & 2764.8 & 133.5 & 188.0 \\
\hline 11 & $(14,5) @(26,1)$ & 1.336 & 14.70 & 2.076 & 1.87 & 124 & 173 & 505.90 & 2849.3 & 124.9 & 174.2 \\
\hline 12 & $(14,7) @(21,10)$ & 1.450 & 19.11 & 2.145 & 18.42 & 127 & 172 & 2899.0 & 2990.8 & 127.3 & 173.2 \\
\hline 13 & $(18,2) @(19,13)$ & 1.494 & 5.21 & 2.182 & 23.82 & 126 & 173 & 3864.0 & 2873.3 & 125.2 & 169.5 \\
\hline 14 & $(18,2) @(20,12)$ & 1.494 & 5.21 & 2.192 & 21.79 & 124.5 & 169 & 3168.1 & 2809.7 & 124.0 & 167.2 \\
\hline 15 & $(13,9) @(24,7)$ & 1.500 & 24.01 & 2.205 & 12.43 & 123 & 168 & 3110.9 & 2576.6 & 122.3 & 165.5 \\
\hline 16 & $(14,8) @(19,14)$ & 1.510 & 21.05 & 2.246 & 25.00 & 121 & 157 & 1253.7 & 3407.8 & 121.3 & 157.7 \\
\hline 17 & $(15,9) @(22,12)$ & 1.644 & 21.79 & 2.338 & 20.36 & 120 & 157 & 3366.7 & 3332.9 & 120.1 & 158.0 \\
\hline 18 & $(18,6) @(21,14)$ & 1.694 & 13.90 & 2.389 & 23.41 & 117.5 & 156 & 3857.3 & 3124.8 & 117.2 & 153.8 \\
\hline 19 & $(16,12) @(27,10)$ & 1.905 & 25.28 & 2.595 & 15.14 & 109 & 141 & 3652.9 & 3067.4 & 109.2 & 143.5 \\
\hline 20 & $(17,14) @(26,14)$ & 2.105 & 26.80 & 2.752 & 20.17 & 105.5 & 148 & 6316.3 & 3850.6 & 105.5 & 148.7 \\
\hline 21 & $(17,14) @(28,12)$ & 2.105 & 26.80 & 2.783 & 17.00 & 101 & 141 & 5418.8 & 2598.9 & 100.9 & 138.5 \\
\hline 22 & $(22,11) @(27,17)$ & 2.278 & 19.11 & 3.009 & 22.52 & 98 & 121 & 3175.3 & 3591.2 & 98.0 & 120.4 \\
\hline
\end{tabular}

parameters could possess slightly different RBLM frequencies. However, on average the $C^{\prime}$ value increases with the increase of outer diameter, and this dependence can be explained as follows.

Assuming no interaction between inner and outer constituent SWCNTs $(G=0)$, the RBM frequency of the outer nanotubes with water molecules deposited on its surface is determined by the upper left $2 \times 2$ part of the matrix (2). Expanding the solution of the quadratic equation derived in such a way within the second order of smallness in $C$, we get

$\omega \approx \sqrt{\frac{4 \gamma}{\rho d_{\text {tube }}^{2}}+\frac{2 C}{\rho d_{\text {tube }}}+\frac{C^{2}}{\left(\gamma \rho_{1}-\gamma_{w} \rho\right)}}$.

Using the substitutions leading from (3) to (4) and the assumption that $C$ is proportional to $d_{\text {tube }}$, one can rewrite the latter expression as

$$
\omega \approx \sqrt{\left(\frac{228}{d_{\text {tube }}}\right)^{2}+C^{\prime}},
$$

where $d_{\text {tube }}=2 R_{2} \approx 2 R_{1}$ and $C^{\prime}=C_{0}^{\prime}+C_{1}^{\prime} d_{\text {tube }}^{2}$. Note that Eq. (5) is reduced to the well-known empirical law ${ }^{54}$ for RBM frequencies $\omega=\left(A / d_{\text {tube }}\right) \operatorname{sqrt}\left(1+c d_{\text {tube }}^{2}\right)$ by substituting $C_{1}^{\prime}=0$. In addition, here we assume that $A=228 \mathrm{~nm} \cdot \mathrm{cm}^{-1}$ and $c=C^{\prime} / A^{2}$.
Let us remark that the dependence of $C^{\prime}$ (or $c$ ) on nanotube's diameter has not been previously discussed, however, the amount of experimental data on SWCNTs ${ }^{23,43,55-61}$ is sufficient to demonstrate it; see the spread of red and blue empty circles in Fig. 2. The $C^{\prime}$ values for DWCNTs presented in Table 1 are plotted depending on outer tubes' diameters (solid blue dots). For these nanotubes, the least-square fit of $C^{\prime}$ values gives $C^{\prime}=$ $2596+82 d_{\text {out }}^{2}$ (blue solid line in Fig. 2). Note that the resulting fit only demonstrates that the $C^{\prime}$ value increases on average with the outer diameter of nanotube. This function is not used when applying the model to find the dependence of $G^{\prime}$ on the structural parameters of DWCNTs.

In the model of coupled oscillators, ${ }^{28}$ both constituent layers of DWCNTs are considered to satisfy the dispersion law $\omega=$ $228 / d$, which is true when no water molecules are adsorbed at the surface of these nanotubes. In order to verify this statement, we have determined the spread of $C^{\prime}$ for the set ${ }^{28}$ of 13 DWCNTs by following the same procedure as for our data within the framework of our model. As shown in Fig. 2, $C^{\prime}$ values are distributed around 0 , as expected. Furthermore, a similar spread in the vicinity of $C^{\prime}=0$ is obtained for SWCNTs ${ }^{62}$ obtained by the same group. Note also that if we substitute $C^{\prime}=0$ into Eq. (4), then the latter one is simplified into the 


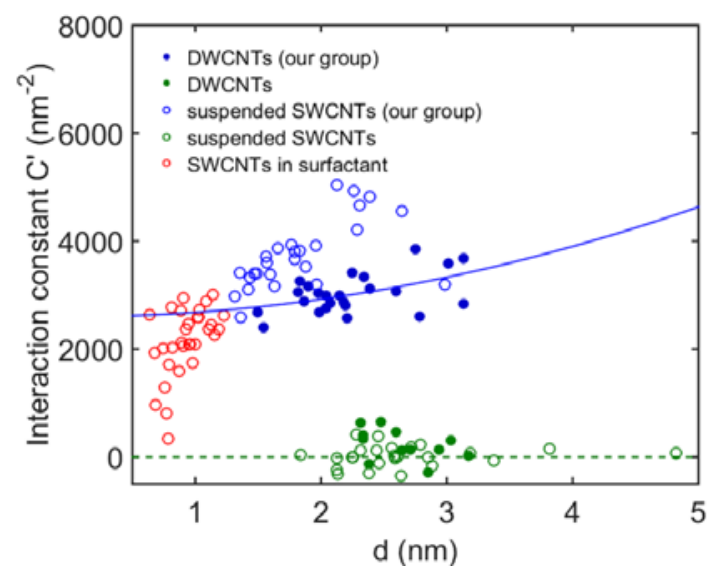

Figure 2. Influence of environment on the dynamics of carbon nanotubes. The dependence of interaction constant $C^{\prime}$ on the outer diameters of suspended indexidentified DWCNTs (from this work: solid blue dots; from Ref. 28: solid green dots) and on the diameters of suspended index-identified SWCNTs (from Ref. 43, 55-61: open blue circles; from Ref. 62: open green circles). Red empty circles denote the spread of $C^{\prime}$ for SWCNTs in surfactant. ${ }^{23}$ The spreads of $C^{\prime}$ for SWCNTs and DWCNTs synthesized in our group (open and solid blue symbols, respectively) are close. A spread around $C^{\prime}=0$ (dashed green line) has been found in suspended DWCNTs ${ }^{28}$ and SWCNTs ${ }^{62}$ synthesized in another group.

equivalent equation ${ }^{50}$ for calculation of coupled oscillators' frequencies.

Let us recall that in the original mode ${ }^{28}$ the interlayer coupling function is approximated by a product between the unit-area force constant, which depends on the interlayer distance only, and the average diameter of a DWCNT. This is justified by the agreement between the force constant and the value of the van der Waals interaction obtained from compressibility measurements of graphite. ${ }^{63}$ The dependence of the unit-area force constant on the interlayer distance in DWCNTs, $\Delta r=\left(d_{\text {out }}-d_{\text {in }}\right) / 2$, can be approximated by a linear expression for $\Delta r$ in the range $0.335-0.37$ $\mathrm{nm} .^{28}$ Following this approximation, we write $G^{\prime}=\left(d_{\text {out }}+\right.$ $\left.d_{\text {in }}\right)\left[L_{0}+L_{1}\left(d_{\text {out }}-d_{\text {in }}\right)\right]$. Then, the least-square fit of data related to DWCNTs ${ }^{28}$ (for which $C^{\prime}=0$ ) yields $L_{0} \approx 7010$ and $L_{1} \approx-8953$ with a standard frequency deviation of $1.30 \mathrm{~cm}^{-1}$. The analogous fit of our data leads to parameters: $L_{0} \approx 8153$ and $L_{1} \approx-10483$ at the standard frequency deviation of $1.39 \mathrm{~cm}^{-1}$. In order to perform this fit, the standard deviation of 48 theoretical RBLM frequencies from the experimental ones was minimized; theoretical frequencies (depended on $L_{0}$ and $L_{1}$ coefficients) were expressed as solutions of 24 Eqs. (4), where the $C^{\prime}$ values were taken from Table 1. The obtained small value of standard deviation justifies that the proposed model of DWCNT dynamics successfully takes into account the environmental influence. However, note that mean diameter of DWCNTs ${ }^{28}$ is larger and, apparently, for this reason the fitting parameters corresponding to the two sets of DWCNTs do not well coincide.

In order to overcome this shortcoming, in the next section we reconsider the explicit form of the interlayer coupling function ${ }^{28}$ and demonstrate that in addition to DWCNT's outer and inner diameters this coupling weakly depends on the twist angle $\Delta \theta$ between nanotube layers. The weakness of the latter chiral term could be seen from Fig. 1c, where RBLM regions of Raman spectra for two index-identified DWCNTs $(12,8) @(16,14)$ and $(16,2) @(16,14)$ are compared. These two tubes have similar mean diameter and interlayer distance, but very different twist angles $\Delta \theta_{h i}\left(4.383^{\circ}\right.$ and $21.978^{\circ}$, respectively, see Table 1$)$. However, in the present case of two DWCNTs with drastically different $\Delta \theta_{h i}$ values not much difference in RBLM frequencies is observed.

\section{Interlayer mechanical coupling with a component dependent on the twist angle}

Study of the twist angle dependent component in interlayer mechanical coupling represents an issue of extreme importance. Recently, analyzing a set of $\sim 70$ nanotubes, authors of Ref. 64 reported that due to the effect of mechanical coupling the formation of DWCNTs with specific helicities is forbidden when $\Delta \theta_{h i} \approx 0^{\circ}$ and $\Delta \theta_{h i}>25^{\circ}$ (in Fig. 3 these are the regions I and II, respectively). Fig. 3 also demonstrates (with open black symbols) the $\left|\theta_{\text {out }}\right|$ vs. $\Delta \theta_{h i}$ values derived from the analysis of 335 DWCNTs synthesized by CCVD, arc-discharge and peapod conversion techniques and described in Refs. 27,28,31,64-79. Apart from these two regions discussed below, there also exists a geometrically forbidden triangular zone (see Fig. 3 ). It is forbidden because $\theta$ is limited in the range $0^{\circ}$ to $30^{\circ}$ and in any graph with $\left|\theta_{\text {out }}\right|$ and $\Delta \theta_{h i}$ axes this zone will be empty even if the values $\theta_{\text {out }}$ and $\theta_{\text {in }}$ are randomly distributed (for more details, see ESI†, S2).

However, in spite of the claim ${ }^{64}$ the helicities presented in Fig. 3 are rather distributed among all possible $\left|\theta_{\text {out }}\right|$ vs. $\Delta \theta_{h i}$ values. In particular, the DWCNT $(14,7) @(21,10)\left(\left|\theta_{\text {out }}\right|\right.$ $\approx 18.42^{\circ}, \Delta \theta_{h i} \approx 0.684^{\circ}$, see Table 1 ) appears in the region I. Moreover, unlike Ref. 64 observations, few DWCNTs $27,28,66,67,72-$ 75 are also located in this region. The upper part of the region II

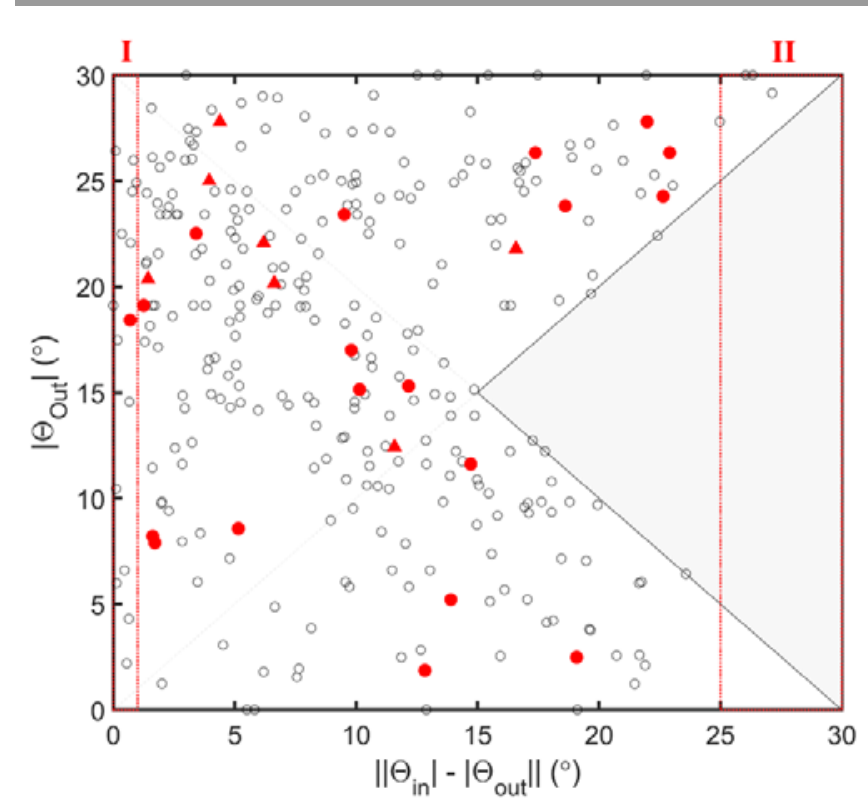

Figure 3. Distribution of 335 helicities for DWCNTs $27,28,31,64-79$ grown by CCVD, arc-discharge and peapod conversion techniques is shown with open black circles; solid red circles and triangles represent the data on our new and earlier reported index-identified individual DWCNTs, respectively. Outlined zones I $\left(\Delta \theta_{h i} \approx 0^{\circ}\right)$ and II $\left(\Delta \theta_{h i}>25^{\circ}\right)$ were claimed in Ref. 64 as forbidden due to the effect of mechanical coupling between the layers.

is also occupied. ${ }^{27,28,74}$ Only the bottom part of the region II 
$\left(\left|\theta_{\text {out }}\right|<5^{\circ}\right)$ is still vacant. Nevertheless, it is not completely correct to say that this small area is prohibited. Only 27 helicities are observed in the strip $\left|\theta_{\text {out }}\right|<5^{\circ}$. If they were random in this strip, then the probability that the small triangular zone with $\Delta \theta_{h i}>25^{\circ}$ appears empty would easily be estimated as $10 \%$. However, the probability (estimated numerically) that a similar small empty zone with the same area exists near a certain $\Delta \theta_{h i}$ value is close to $100 \%$. Thus, the plausible hypothesis that the interlayer mechanical coupling prevents the formation of the considered helicities is not yet justified experimentally. However, we support the idea ${ }^{64}$ that this coupling could depend on the value of the twist angle and try to evaluate below the weak dependence of the coupling constant $G^{\prime}$ on the value of $\Delta \theta$.

Let us consider how the constant of elastic coupling between two flat graphene sheets changes if they are mutually rotated by the twist angle $\Delta \theta=\theta_{\text {in }}-\theta_{\text {out }}$ forming so-called moiré patterns, actively discussed in literature. ${ }^{27,30}$ First, if at $\Delta \theta=0$ the orientations of sheets coincide, then at $\Delta \theta=60^{\circ}$ they coincide again. Second, the constants of elastic coupling should be equal at any twist angles $\Delta \theta$ and $-\Delta \theta$. Therefore, a dependence of elastic coupling of two flat graphene sheets on $\Delta \theta$ can be presented as a series with basic functions $f_{n}=\cos (6 n \Delta \theta)$, where $n$ are nonnegative integers (see similar symmetricallyjustified consideration in Ref. 80). The simplest form for this dependence (not reduced to a constant) is $\cos (6 \Delta \theta)$ and we will use it below to model angular anisotropy of interlayer coupling in DWCNTs.

In the original model ${ }^{28}$ of coupled oscillators the interlayer coupling $G^{\prime}$ is a quadratic function of nanotube's diameters $d_{\text {out }}$ and $d_{i n}$. However, at some finite range any function without extreme points can be approximated by a linear one. So we choose the following simplest form for $G^{\prime}$ :

$$
\begin{aligned}
G^{\prime}=G_{0}+G_{1}\left(d_{\text {out }}\right. & \left.-d_{\text {in }}\right)+G_{2}\left(d_{\text {out }}+d_{\text {in }}\right) \\
& +\quad G_{3}\left(d_{\text {out }}+d_{\text {in }}\right) \cos (6 \Delta \theta),
\end{aligned}
$$

where four coefficients $G_{0} \div G_{3}$ are the constants, which do not depend on structural parameters of DWCNTs. The first three achiral terms in (6) correspond to the general linear function in $d_{\text {out }}$ and $d_{\text {in }}$, which were grouped to emphasize the contributions proportional to the interlayer distance and the average diameter of DWCNT. The fourth term (containing $\Delta \theta$ ) for the sake of simplicity is assumed to depend only on the DWCNT's average diameter.

For the practical utilization of Eq. (6) we replace $\Delta \theta=\theta_{\text {in }}$ $\theta_{\text {out }}$ with $\Delta \theta_{\text {hi }}$ (see a justification for this replacement in the next section) and determine $G_{0} \div G_{3}$ constants in two stages for convenience. First, we find their approximate values by minimizing the standard deviation of the function (6) from the $G^{\prime}$ values presented in Table 1 . This problem is reduced to the system of linear equations which has a unique solution. At the second stage, the standard deviation of 48 theoretical RBLM frequencies from the experimental ones is minimized. Theoretical frequencies depend on $G_{0} \div G_{3}$ coefficients and are expressed as solutions of 24 Eqs. (4), where we utilize the previously found values of $C^{\prime}$ (see Table 1). In order to perform the final minimization, $G_{i}$ constants found at the first stage are used as a first approximation. As a result, we get

$$
G_{0} \approx 31644, G_{1} \approx-45839, G_{2} \approx 882, G_{3} \approx 49 .
$$

The use of Eq. (4), where $C^{\prime}$ is taken from Table 1 and $G^{\prime}$ is calculated in terms of (6) with coefficients (7) leads to theoretical RBLM frequencies $\omega_{L}^{\text {theor }}$ and $\omega_{H}^{\text {theor }}$ presented in the last two columns of Table 1 (see also ESI†, S3). The maximum deviation of theoretical frequencies from the experimental ones does not exceed $3.5 \mathrm{~cm}^{-1}$ (DWCNT \#13 $(18,2) @(19,13))$, and the standard deviation decreased to 1.27 $\mathrm{cm}^{-1}$. Thus, the numerical analysis described above supports both the semi-empirical formula (6) and the presented model of RBLM dynamics.

After that we first utilize the $G^{\prime}$ dependence (6) with the coefficients (7) found by fitting our data and obtain theoretical frequencies $\omega_{L}^{\text {theor }}$ and $\omega_{H}^{\text {theor }}$ for 13 DWCNTs presented in the Ref. 28. For this calculation, the simplification $C^{\prime}=0$ in Eq. (4) is used and the maximum deviation between theoretical and experimental frequencies does not exceed $2.7 \mathrm{~cm}^{-1}$ (DWCNT $(15,10) @(27,6))$, while the standard deviation is equal to 1.6 $\mathrm{cm}^{-1}$ (see also ESIt, S3). Thus, the formula (6) with the coefficients (7) is in good agreement with the data taken from literature as well. Second, we perform the direct numerical minimization of standard frequency deviation for these 13 DWCNTs. It leads to slightly different coefficients in formula (6) as compared to those found for our DWCNTs:

$$
G_{0} \approx 31680, G_{1} \approx-46664, G_{2} \approx 948, G_{3} \approx 46 .
$$

For this fit we find that the maximum deviation between theoretical and experimental frequencies increases to $3 \mathrm{~cm}^{-1}$ (DWCNT $(14,12) @(23,13))$, yet standard deviation goes down to 1.20 $\mathrm{cm}^{-1}$. Let us also note that the formula (6) with coefficients (8) leads to standard deviation of $1.4 \mathrm{~cm}^{-1}$ for the DWCNTs from Table 1 . Thus, we can conclude that both sets (7) and (8) of $G_{i}$ parameters are appropriate for all DWCNTs considered in this paper. The relation (6) seems to be more robust because it concurrently fits both sets of data with smaller standard deviations (in respect to those calculated in the previous section). Overall, the relation (6) represents a mathematically justified approximation of interlayer interaction, the physical mechanism of which needs to be clarified in further studies.

\section{Angular dependence of interlayer coupling and peculiarities of self-assembly of DWCNTs}

The majority of the modern methods of DWCNT nanoidentification (including the ones used for indexing of the DWCNTs listed in Table 1 and Ref. 28) do not distinguish between left- and right-handed nanotubes in a pair $(n, m) @(p, q)$ and $(m, n) @(q, p)$. Moreover, they do not differentiate a DWCNT $(n, m) @(p, q)$ from the ones $(n, m) @(q, p)$ and $(m, n) @(p, q)$. Thus, if for two SWCNTs, which form a DWCNT, chiral angles are found as nonnegative values $\theta_{\text {in }}$ and $\theta_{\text {out }}$ less than $30^{\circ}$, then in a DWCNT comprising the layers of opposite handedness the value $|\Delta \theta|$ appears to be equal not to the difference between moduli of chiral angles, but to their sum. Fig. 4a illustrates the case when $|\Delta \theta|=\left|\theta_{\text {in }}\right|+\left|-\theta_{\text {out }}\right|$ and explains how we determine the sign of chiral angles. An alternative (but totally 
equivalent) definition can be found in Ref. 8 but appears less convenient to us.

Since $|\Delta \theta|$ may be equal not only to the difference between moduli of chiral angles but also to their sum, it may seem that the presence of the term proportional to $\cos (6 \Delta \theta)$ in Eq. (6) is meaningless, because these two cases lead to different values of $\cos (6 \Delta \theta)$. In order to clarify this fact, let us present $\cos (6 \Delta \theta)$ as $\cos \left(6 \theta_{\text {in }}\right) \cos \left(6 \theta_{\text {out }}\right)+\sin \left(6 \theta_{\text {in }}\right) \sin \left(6 \theta_{\text {out }}\right)$. The first term of the latter equation does not depend on handedness of the SWCNTs forming the DWCNT, while the second term changes its sign if one of the layers is replaced by its mirror-symmetrical copy. Thus, if the formation of all four considered configurations of DWCNTs was equally probable, then $\cos (6 \Delta \theta)$ in Eq. (6) should be replaced with the handedness-invariant product $\cos \left(6 \theta_{\text {in }}\right) \cos \left(6 \theta_{\text {out }}\right)$.

Having performed this replacement and used the fitting procedure described above, we recalculated coefficients (7-8) and found new values of standard deviations, which increased by 0.2 and $0.3 \mathrm{~cm}^{-1}$, respectively. Since fitting with the initial function (6) turns out to give the best results, we believe that a DWCNT rather comprises the layers with the same handedness. Our opinion is supported by the well-known results that SWCNTs align on the graphite substrate according to their handedness ${ }^{11}$ or that the adsorption of complex chiral molecules on the SWCNT surface occurs when their handedness fit that of the tube. ${ }^{20}$

\section{How the results obtained can be used for the verification of DWCNT identification?}

First, the method described below is reliable if structural parameters of a considered DWCNT belong to the interval of parameters of DWCNTs discussed in the present work. In particular, the mean diameter $\left(d_{\text {out }}+d_{\text {in }}\right) / 2$ should be approximately in the range 1-3 $\mathrm{nm}$. At such mean diameters, for different DWCNTs the contribution of $G_{1}\left(d_{\text {out }}-d_{\text {in }}\right)$ to the value of interlayer coupling (6) can vary with a dispersion of about 3000 units, while the analogous dispersion for the chiral term proportional to $G_{3}$ is approximately 6 times smaller. However, since close values of $G_{3}$ are obtained when performing analysis of DWCNTs from different sources, the addition of this term is reasonable. It decreases the maximal deviations between theoretical and experimental frequencies by $\approx 1 \mathrm{~cm}^{-1}$ that, in turn, makes our approach substantially more accurate for determination of errors in identification of DWCNTs.

If a considered DWCNT is in air of some nonzero humidity, at first one should find coefficients $C^{\prime}$ and $G^{\prime}$ using its RBLM frequencies and structural parameters (this procedure is described below Eq. (4)). If the latter coefficient differs significantly (by more than 500-600 units) from the result of calculation by means of Eq. (6), then a detailed analysis should be performed in a following way. First, replace your coefficient $G^{\prime}$ with the result of its calculation by means of Eq. (6). Second, use Eq. (4) in order to find theoretical RBLM frequencies. If for at least one of the modes the difference between theoretical and experimental RBLM frequencies exceeds 5-6 $\mathrm{cm}^{-1}$, then there is a strong probability that an error of identification of the DWCNT has occurred.
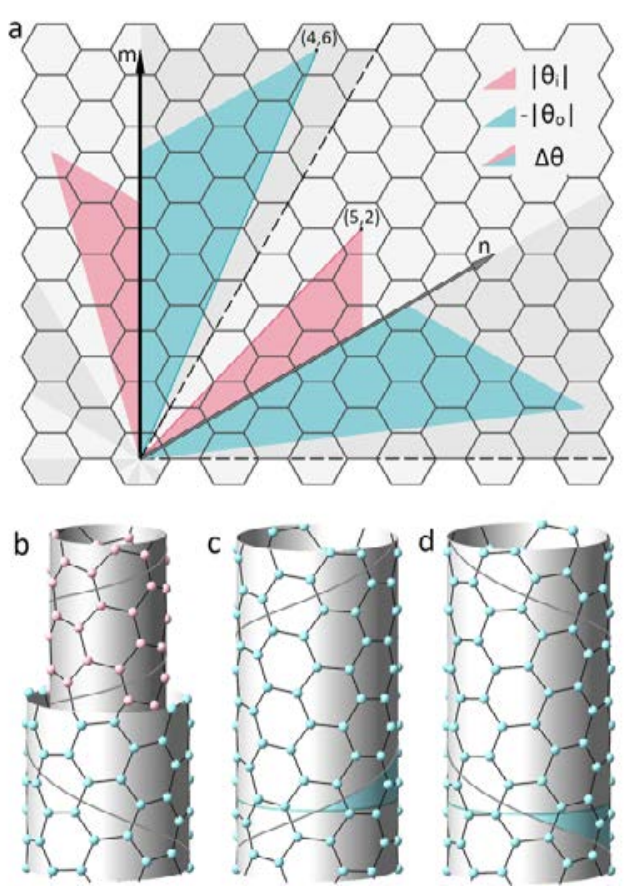

Figure 4. The handedness of layers and the twist angle $\Delta \theta$ in DWCNTs.(a) Chiral vectors and chiral angles for the DWCNT comprising two layers of opposite handedness. The planar symmetry of a graphene sheet is described by the group $C_{6 v}$. Therefore, without taking into account the handedness, any SWCNT is indexed by a chiral vector, which becomes a perimeter of the nanotube and lays within one $30^{\circ}$ fundamental sector between two neighboring mirror planes of $C_{6 v}$ symmetry group. ${ }^{8}$ Alternate neighboring fundamental sectors are shown in panel (a) in light and dark hues. Taking into account the handedness, one should use any two neighboring sectors separated by a mirror plane of $C_{6 v}$ symmetry group for SWCNT indexing. Then chiral vectors generating left- and right-handed antisymmetic SWCNTs are reflected in each other by this mirror plane, and chiral vectors rotated by $60^{\circ}$ give rise to identical SWCNTs. In order to index SWCNTs, we choose two sectors separated by $\mathrm{n}$ axis and assume that a chiral angle $\theta$ is measured in clockwise and anticlockwise directions with respect to the vector $\mathrm{n}$ and $-30^{\circ} \leq \theta \leq 30^{\circ}$. As it is shown, due to the opposite handedness of layers in the DWCNT considered,

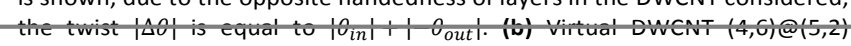
comprising two layers of opposite handedness. (c-d) Pair $(6,4)$ and $(4,6)$ of SWCNTs with opposite handedness. The change of indices from $(n, m)$ to $(m, n)$ is always equivalent to the change of handedness. Colored sectors correspond to chiral angles and lie between chiral vectors (transformed into the tube perimeters) and former directions (zigzag) of $n$ axis shown in panel (a).

If you work in vacuum (or at zero humidity), then substitute $C^{\prime}=0$ and $G^{\prime}$ found by means of Eq. (6) in Eq. (4) and solve it. Once again, if for at least one of the modes the difference between theoretical and experimental RBLM frequencies exceeds 5-6 $\mathrm{cm}^{-1}$, then there is a strong probability that an error of identification of the DWCNT has occurred.

Note that it is possible to use any set of coefficients ((7) or (8)) in formula (6). In spite of the fact that the set (7) is more appropriate for DWCNTs in humid air while the set (8) is better at zero humidity, the arising difference in determination of errors would be unessential. In future, if the dependence of $G^{\prime}$ on structural parameters of DWCNTs is defined more precisely, our approach can be used not only for determination of errors in DWCNT identification, but also for the procedure of identification itself. Even now use of Eqs. (4) and (6) with coefficients (7) has allowed us to identify the structures of 3 DWCNTs not listed in Table 1 (see ESI†, S4). This identification would have been impossible without the results presented above. 


\section{Conclusions}

In the present work, on the basis of our new experimental results and experimental data published previously, ${ }^{28,29,45}$ we consider how chirality and handedness of walls forming a DWCNT manifest themselves in the dynamics of its phonon breathing-like modes. The proposed symmetrically-justified model turns out to be substantially more accurate than the previous ones since within its framework we have succeeded not only to take into account a possible deposition of water molecules onto an outer DWCNT wall from ambient air but also to determine the contribution from the handedness-dependent term in interlayer mechanical coupling between walls forming the DWCNT. Statistical evidence that this term has an influence on RBLM dynamics allows us to draw the following inference: the formation of DWCNTs consisting of the layers with the same handedness is more probable. This statement is in accordance with the known recent papers demonstrating that SWCNT aligns on the graphite substrate according to its handedness ${ }^{11}$ or that for the adsorption of complex chiral molecules (DNA, for example), the handedness of them should fit that of the SWCNT, 20 to the surface of which the molecules are attached. DWCNTs with specific handedness of layers could be functionalized with different chiral bioactive molecules and could be used to design hybrid CNT-based nanobioelectronic devices $^{81}$ and biomedicine tools $\mathrm{s}^{82}$ that is of great interest for both interdisciplinary research and future applications. Furthermore, from a technological viewpoint, our results could become the basis for a new method of DWCNT identification, which would use the established dependence of RBLM frequencies on structural parameters (including chiral angles) of DWCNTs. The paper will be of interest to a wide range of readers dealing with synthesis, phonon dynamics, identification, as well as structural and symmetry peculiarities of carbon nanotubes.

\section{Methods}

\section{Growth of individual DWCNTs on open slit substrates}

Ultralong individual DWCNTs were grown by catalytic chemical vapor deposition (CCVD) method directly across open slit structures $\left(\sim 50 \times 1000 \mu \mathrm{m}^{2}\right)$ fabricated on silicon (Si) substrates ((100) orientation, $200 \mu \mathrm{m}$ thick) with a $90 \mathrm{~nm}$ thick $\mathrm{SiO}_{2}$ layer (as shown on Figure 1a). $0.001 \mathrm{M}$ of iron (III) chloride $\left(\mathrm{FeCl}_{3}\right)$ solution was used as catalyst precursor, deposited on $\mathrm{SiO}_{2} / \mathrm{Si}$ substrates. During the CCVD, the catalyst substrates were put side by side with the slit substrates. The substrates were oriented with the catalyst side towards the gas inlet. The sample held in a quartz tube was kept outside the heated zone during temperature ramping of the furnace. Before heating, all gas lines and the reactor tube were purged by argon (Ar) at a flow rate of $800 \mathrm{sccm}$ for $30 \mathrm{~min}$. Next, the furnace was heated to $950{ }^{\circ} \mathrm{C}$ at a ramping rate of $18.5^{\circ} \mathrm{C} \mathrm{min}^{-1}$. To carry ethanol $\left(\mathrm{C}_{2} \mathrm{H}_{5} \mathrm{OH}\right)$ to the furnace, $\mathrm{Ar}$ at a flow of $15 \mathrm{sccm}$ was passed through a bubbler containing $\mathrm{C}_{2} \mathrm{H}_{5} \mathrm{OH}$ (kept at room temperature $\sim 25{ }^{\circ} \mathrm{C}$ ). When the temperature reached the synthesis temperature, $\operatorname{Ar}$ was replaced by hydrogen $\left(\mathrm{H}_{2}\right)$ and $\mathrm{C}_{2} \mathrm{H}_{5} \mathrm{OH} / \mathrm{Ar}$ at flow rates (1:1 ratio) of $15 \mathrm{sccm}$. After $5 \mathrm{~min}$, the furnace was slid to the sample position to fast heat the sample and to grow CNTs. The synthesis durations were $30 \mathrm{~min}$. After that, $\mathrm{H}_{2}$ and $\mathrm{C}_{2} \mathrm{H}_{5} \mathrm{OH}$ vapor were replaced by pure $\mathrm{Ar}$ at a flow rate of $30 \mathrm{sccm}$, the furnace was switched off and was slid to the initial position to fast cool the samples.

\section{Measurements}

High resolution transmission electron microscopy (HRTEM) images and electron diffraction (ED) patterns were recorded in a FEI Titan Cube microscope (equipped with a spherical aberration corrector) operating at $80 \mathrm{kV}$ and within short acquisition times (less than $5 \mathrm{~s}$ for ED) to reduce damage induced by electron diffraction. Rayleigh spectra of individual free-standing DWCNTs were measured in a backscattering geometry using a cross-polarization scheme with a Fianium supercontinuum laser as a light source and a fiber-fitted QE-Pro Ocean Optics spectrometer for detection. Resonant Raman scattering measurements were carried out using an iHR550 Jobin-Yvon spectrometer (gratings 1,800 or 1,200 grooves per $\mathrm{mm}$ ) equipped with a liquid-nitrogen-cooled, back-illuminated silicon charge-coupled device detector in a micro Raman backscattering configuration. The home-made microscope was equipped with $a \times 100$ objective (numerical aperture $=0.95$ ). The samples were mounted on a three-axis piezo-electric stage (PIMars P-563, Physik Instrumente) to ensure the precise positioning and focusing of the laser spot. Incident excitations from various continuous wave lasers were used: $\mathrm{HeNe}$ at 632.8 $\mathrm{nm}(1.96 \mathrm{eV})$, diode-pumped solid-state lasers at $457 \mathrm{~nm}$ (2.71 $\mathrm{eV}), 532 \mathrm{~nm}(2.33 \mathrm{eV})$ and $561 \mathrm{~nm}(2.21 \mathrm{eV})$, and a Ti-Sapphire filtered using a tunable laser line filter in the near infrared. To avoid heating effects, the laser intensity impinging the sample was kept below $100 \mathrm{~kW} / \mathrm{cm}^{2}$.

\section{Author Contributions}

S.R. with the essential help of D.L conceived of the main theoretical ideas developed in this paper. D.L. performed also the statistical analysis of DWCNTs. S.R. with the help of D.L., M.A., J.-L. S., M.P. wrote the main part of the text. Experimental data were obtained by D.L., R.A., T.T.C, V.C.N., J.-L. S., M.P. All authors participated in discussion and writing of the article.

\section{Conflicts of interest}

The authors declare that they have no competing interests.

\section{Acknowledgements}

Authors are grateful to Olga Konevtsova, who has designed the Graphical Abstract and Fig. 4. S.R., D.L. and M.A acknowledge financial support from the Russian Foundation for Basic Research (grant № 18-29-19043 mk). HRTEM and ED studies were conducted at the Laboratorio de Microscopias Avanzadas, Instituto de Nanociencia de Aragon, Universidad de Zaragoza, Spain. The Government of Aragon, and the European Social Fund are gratefully acknowledged. R.A. gratefully acknowledges the project "Construyendo Europa desde Aragon" 2014-2020 (grant number E13/17R). R.A. gratefully acknowledges the support from the Spanish Ministry of Economy and 
Competitiveness (MINECO) through project grant MAT201679776-P (AEI/FEDER, UE).

\section{Notes and references}

1 S. lijima, Nature, 1991, 354, 56-58.

2 A. D. Franklin, Science, 2015, 349, 6249.

3 M. M. Shulaker, G. Hills, N. Patil, H. Wei, H. Y. Chen, H. S. P. Wong and S. Mitra, Nature, 2013, 501, 526-530.

4 M. M. Shulaker, G. Hills, R. S. Park, R. T. Howe, K. Saraswat, H. S. P. Wong and S. Mitra, Nature, 2017, 547, 74-78.

5 G. D. Ado Jorio, M. S. Dresselhaus, A. Jorio, M. S. Dresselhaus, G. D. Ado Jorio and M. S. Dresselhaus, Mater. Today, 2008, 11, 57.

6 S. Reich, C. Thomsen and J. Maultzsch, Basic Concepts and Physical Properties, WILEY-VCH Verlag GmbH \& Co. KGaA, 1st edn., 2004.

7 A. Loiseau, P. Launois, P. Petit, S. Roche and J.-P. Salvetat, Nano Today, 2006, 1, 51.

8 G. G. Samsonidze, A. Grüneis, R. Saito, A. Jorio, A. G. Souza Filho, G. Dresselhaus and M. S. Dresselhaus, Phys. Rev. B Condens. Matter Mater. Phys., 2004, 69, 205402.

9 C. A. Badalucco, J. A. Gascón, F. Papadimitrakopoulos, D. C. Abanulo and S.-Y. Ju, J. Am. Chem. Soc., 2012, 134, 1319613199.

10 A. I. A. Abd El-Mageed, T. Inose, Z. Chen, T. Ogawa and M. Handayani, Chem. - A Eur. J., 2018, 25, 1941-1948.

11 Y. Chen, Z. Shen, Z. Xu, Y. Hu, H. Xu, S. Wang, X. Guo, Y. Zhang, L. Peng, F. Ding, Z. Liu and J. Zhang, Nat. Commun., 2013, 4, 2205.

12 V. Mallouh, P. Schultz, C. Mioskowski, T. W. Ebbesen, C. Richard and F. Balavoine, Angew. Chemie Int. Ed., 2002, 38, 1912-1915.

13 N. W. S. Kam, T. C. Jessop, P. A. Wender and H. Dai, J. Am. Chem. Soc., 2004, 126, 6850-6851.

14 G. Ghirlanda, J. D. Lear, A. Lombardi and W. F. Degrado, J. Mol. Biol., 1998, 281, 379-391.

15 J. Reina, E. Lacroix, S. D. Hobson, G. Fernandez-Ballester, V Rybin, M. S. Schwab, L. Serrano and C. Gonzalez, Nat. Struct. Biol., 2002, 9, 621-627.

16 G. R. Dieckmann, A. B. Dalton, P. A. Johnson, J. Razal, J. Chen, G. M. Giordano, E. Muñoz, I. H. Musselman, R. H. Baughman and R. K. Draper, J. Am. Chem. Soc., 2003, 125, 1770-1777.

17 G. Grigoryan, A. W. Reinke and A. E. Keating, Nature, 2009, 458, 859-864.

18 J.-S. Kim and C. O. Pabo, Proc. Natl. Acad. Sci., 2002, 95, 28122817.

19 J. Ashworth, J. J. Havranek, C. M. Duarte, D. Sussman, R. J. Monnat, B. L. Stoddard and D. Baker, Nature, 2006, 441, 656659.

20 G. Ao, J. K. Streit, J. A. Fagan and M. Zheng, J. Am. Chem. Soc., 2016, 138, 16677-16685.

21 M. F. L. De Volder, S. H. Tawfick, R. H. Baughman and A. J. Hart, Science, 2013, 339, 535-539.

22 M. Steiner, M. Freitag, J. C. Tsang, V. Perebeinos, A. A. Bol, A V. Failla and P. Avouris, Appl. Phys. A Mater. Sci. Process., 2009, 96, 271-282.

23 S. Cambré, B. Schoeters, S. Luyckx, E. Goovaerts and W. Wenseleers, Phys. Rev. Lett., 2010, 104, 207401.

24 C. Shen, A. H. Brozena and Y. Wang, Nanoscale, 2011, 3, 503518.

25 R. Pfeiffer, T. Pichler, Y. A. Kim and H. Kuzmany, in Springer Series on Topics in Appl. Phys., 2008, 111, pp. 495-530.

26 K. Fujisawa, H. J. Kim, S. H. Go, H. Muramatsu, T. Hayashi, M. Endo, T. C. Hirschmann, M. S. Dresselhaus, Y. A. Kim and P. T. Araujo, Appl. Sci., 2016, 6, 109.

27 K. Liu, C. Jin, X. Hong, J. Kim, A. Zettl, E. Wang and F. Wang, Nat. Phys., 2014, 10, 737-742.
28 K. Liu, X. Hong, M. Wu, F. Xiao, W. Wang, X. Bai, J. W. Ager, S. Aloni, A. Zettl, E. Wang and F. Wang, Nat. Commun., 2013, 4, 1375.

29 D. Levshov, T. X. Than, R. Arenal, V. N. Popov, R. Parret, M. Paillet, V. Jourdain, A. A. Zahab, T. Michel, Y. I. Yuzyuk and J.L. L. Sauvajol, Nano Lett., 2011, 11, 4800-4804.

30 M. Koshino, P. Moon and Y. W. Son, Phys. Rev. B - Condens. Matter Mater. Phys., 2015, 91, 035405.

31 O. Postupna, R. Long and O. V. Prezhdo, J. Phys. Chem. C, 2015, 119, 12088-12094.

32 C. S. Allen, A. W. Robertson, A. I. Kirkland and J. H. Warner, Small, 2012, 8, 3810-3815.

33 J. W. G. Wildöer, L. C. Venema, A. G. Rinzler, R. E. Smalley and C. Dekker, Nature, 1998, 391, 59-62.

34 L. C. Qin, Phys. Chem. Chem. Phys., 2007, 9, 31-48.

35 S. Amelinckx, A. Lucas and P. Lambin, Reports Prog. Phys. 1999, 62, 1471-1524.

36 S. M. Bachilo, M. S. Strano, C. Kittrell, R. H. Hauge, R. E. Smalley and R. B. Weisman, Science, 2002, 298, 2361-2366.

37 M. Y. Sfeir, F. Wang, L. Huang, C. C. Chuang, J. Hone, S. P. O'Brien, T. F. Heinz and L. E. Brus, Science, 2004, 306, 15401543.

38 J. C. Blancon, M. Paillet, H. N. Tran, X. T. Than, S. A. Guebrou, A. Ayari, A. S. Miguel, N. M. Phan, A. A. Zahab, J. L. Sauvajol, N. Del Fatti and F. Vallée, Nat. Commun., 2013, 4, 2542.

39 A. Jorio, C. Fantini, M. A. Pimenta, R. B. Capaz, G. G. Samsonidze, G. Dresselhaus, M. S. Dresselhaus, J. Jiang, N. Kobayashi, A. Grüneis and R. Saito, Phys. Rev. B - Condens. Matter Mater. Phys., 2005, 71, 075401.

40 H. Telg, J. Maultzsch, S. Reich, F. Hennrich and C. Thomsen, Phys. Rev. Lett., 2004, 93, 177401.

41 C. Fantini, A. Jorio, M. Souza, M. S. Strano, M. S. Dresselhaus and M. A. Pimenta, Phys. Rev. Lett., 2004, 93, 147406.

42 R. Saito, M. Hofmann, G. Dresselhaus, A. Jorio and M. S. Dresselhaus, Adv. Phys., 2011, 60, 413-550.

43 T. Michel, D. Levshov, A.-A. Zahab, J.-L. Sauvajol and M. Paillet, in Handbook of Carbon Nanomaterials Vol. 10: Optical Properties of Carbon Nanotubes, eds. R. B. Weisman and J. Kono, World Scientific Publishing, Singapore, 2019, pp. 75112.

44 T. Michel, M. Paillet, D. Nakabayashi, M. Picher, V. Jourdain, J. C. Meyer, A. A. Zahab and J. L. Sauvajol, Phys. Rev. B - Condens. Matter Mater. Phys., 2009, 80, 245416.

45 D. I. Levshov, H. N. Tran, M. Paillet, R. Arenal, X. T. Than, A. A. Zahab, Y. I. Yuzyuk, J. L. Sauvajol and T. Michel, Carbon, 2017, 114, 141-159.

46 S. Chiashi, K. Kono, D. Matsumoto, J. Shitaba, N. Homma, A. Beniya, T. Yamamoto and Y. Homma, Phys. Rev. B - Condens. Matter Mater. Phys., 2015, 91, 1-5.

47 T. X. Tinh, N. V. Chuc, V. Jourdain, M. Paillet, D. Y. Kim, J. L. Sauvajol, N. T. T. Tam and P. N. Minh, J. Exp. Nanosci., 2011, 6, 547-556.

48 C. S. Allen, C. Zhang, G. Burnell, A. P. Brown, J. Robertson and B. J. Hickey, Carbon N. Y., 2011, 49, 4961-4971.

49 D. I. Levshov, R. Parret, H.-N. Tran, T. Michel, T. T. Cao, V. C. Nguyen, R. Arenal, V. N. Popov, S. B. Rochal, J.-L. Sauvajol, A.A. Zahab and M. Paillet, Phys. Rev. B, 2017, 96, 195410.

50 S. B. Rochal, V. L. Lorman and Y. I. Yuzyuk, Phys. Rev. B Condens. Matter Mater. Phys., 2013, 88, 235435.

51 D. I. Levshov, M. V Avramenko, X.-T. Than, T. Michel, R. Arenal, M. Paillet, D. V. Rybkovskiy, A. V Osadchy, S. B. Rochal, Y. I. Yuzyuk and J. Sauvajol, J. Nanophotonics, 2015, 10, 012502.

52 D. Levshov, T. Michel, M. Paillet, X. T. Than, H. N. Tran, R. Arenal, A. Rahmani, M. Boutahir, A.-A. Zahab and J. L. Sauvajol, MRS Online Proceedings Library Archive, 2014, 1700, 69-77.

53 J. Yang, D. Zhang, Y. Hu, C. Xia, S. Sun and Y. Li, ACS Nano, 2017, 11, 10509-10518. 
54 P. Araujo, I. Maciel, P. Pesce, M. Pimenta, S. Doorn, H. Qian, A. Hartschuh, M. Steiner, L. Grigorian, K. Hata and A. Jorio, Phys. Rev. B, 2008, 77, 241403.

55 M. Paillet, T. Michel, A. Zahab, D. Nakabayashi, V. Jourdain, R. Parret, J. Meyer and J. L. Sauvajol, Phys. Status Solidi Basic Res., 2010, 247, 2762-2767.

56 D. Levshov, T. Michel, T. Than, M. Paillet, R. Arenal, V. Jourdain, Y. I. Yuzyuk and J.-L. Sauvajol, J. Nanoelectron. Optoelectron., 2013, 8, 9-15.

57 M. Paillet, T. Michel, J. C. Meyer, V. N. Popov, L. Henrard, S. Roth and J. L. Sauvajol, Phys. Rev. Lett., 2006, 96, 257401.

58 J. C. Meyer, M. Paillet, T. Michel, A. Moréac, A. Neumann, G. S. Duesberg, S. Roth and J. L. Sauvajol, Phys. Rev. Lett., 2005, 95, 217401

59 T. Michel, M. Paille, J. C. Meyer, V. N. Popov, L. Henrard, P. Poncharal, A. Zahab and J. L. Sauvajol, in Physica Status Solidi (B) Basic Research, 2007, 244, 3986-3991.

60 A. Débarre, M. Kobylko, A. M. Bonnot, A. Richard, V. N. Popov L. Henrard and M. Kociak, Phys. Rev. Lett., 2008, 101, 197403.

61 A. A. Tonkikh, S. B. Roshal', D. I. Levshov, T. Michel, Y. S. Slabodyan and Y. I. Yuzyuk, Phys. Solid State, 2017, 59, 594600.

62 K. Liu, W. Wang, M. Wu, F. Xiao, X. Hong, S. Aloni, X. Bai, E. Wang and F. Wang, Phys. Rev. B - Condens. Matter Mater. Phys., 2011, 83, 113404.

63 M. Hanfland, H. Beister, and K. Syassen, Phys. Rev. B, 1989, 39, 12598-12603.

64 A. Ghedjatti, Y. Magnin, F. Fossard, G. Wang, H. Amara, E. Flahaut, J. S. Lauret and A. Loiseau, ACS Nano, 2017, 11, 48404847.

65 A. Hashimoto, K. Suenaga, K. Urita, T. Shimada, T. Sugai, S. Bandow, H. Shinohara and S. lijima, Phys. Rev. Lett., 2005, 94, $1-4$.

66 K. Hirahara, M. Kociak, S. Bandow, T. Nakahira, K. Itoh, Y. Saito and S. lijima, Phys. Rev. B - Condens. Matter Mater. Phys. 2006, 73, 195420.

67 S. Zhao, T. Kitagawa, Y. Miyauchi, K. Matsuda, H. Shinohara and R. Kitaura, Nano Res., 2014, 7, 1548-1555.

68 M. Kociak, K. Suenaga, K. Hirahara, Y. Saito, T. Nakahira and S. lijima, Phys. Rev. Lett., 2002, 89, 155501/1-155501/4.

69 M. Kociak, K. Hirahara, K. Suenaga and S. lijima, Eur. Phys. J. B, 2003, 32, 457-469.

70 J. M. Zuo, I. Vartanyants, M. Gao, R. Zhang and L. A. Nagahara, Science, 2003, 300, 1419-1421.

71 J. M. Zuo, T. Kim, A. Celik-Aktas and J. Tao, Zeitschrift fur Krist., 2007, 222, 625-633.

72 M. Gao, J. M. Zuo, R. Zhang and L. A. Nagahara, Journal of Materials Science, 2006, 41, 4382-4388.

73 J. F. Colomer, L. Henrard, P. Launois, G. Van Tendeloo, A. A. Lucas and P. Lambin, Chem. Commun., 2004, 10, 2592-2593.

74 K. Liu, Z. Xu, W. Wang, P. Gao, W. Fu, X. Bai and E. Wang, J. Phys. D. Appl. Phys., 2009, 42, 125412.

75 J. F. Colomer, L. Henrard, P. Launois, G. Van Tendeloo, A. A. Lucas and P. Lambin, Phys. Rev. B - Condens. Matter Mater. Phys., 2004, 70, 075408.

76 H. Zhu, K. Suenaga, A. Hashimoto, K. Urita and S. lijima, Chem. Phys. Lett., 2005, 412, 116-120.

77 H. Zhu, K. Suenaga, J. Wei, K. Wang and D. Wu, J. Phys. Chem. C, 2008, 112, 11098-11101.

78 L. Guan, K. Suenaga and S. lijima, Nano Lett., 2008, 8, 459462.

79 J. F. Colomer, L. Henrard, G. V. Tendeloo, A. Lucas and P. Lambin, J. Mater. Chem., 2004, 14, 603-606.

80 F. Ye, B. S. Wang and Z. Bin Su, Phys. Rev. B - Condens. Matter Mater. Phys., 2006, 73, 1-5.

81 E. Katz and I. Willner, ChemPhysChem, 2004, 5, 1084-1104.

82 C. Ménard-Moyon, K. Kostarelos, M. Prato and A. Bianco, Chem. Biol., 2010, 17, 107-115. 